\title{
Diagnostic role of neutrophil-to-lymphocyte ratio and monocyte-to-lymphocyte ratio in patients with enchondroma and low-grade chondrosarcoma
}

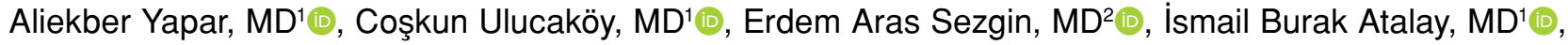 \\ Mehmet Fatih Ekşioğlu, MD¹i \\ 'Department of Orthopedics and Traumatology, Dr. Abdurrahman Yurtaslan Ankara Oncology Training and Research Hospital, Ankara, Turkey \\ ${ }^{2}$ Department of Orthopaedics and Traumatology, Aksaray University, Training and Research Hospital, Aksaray, Turkey
}

Cartilaginous tumors of the skeleton are amongst the most common; however, differential diagnosis remains to be a challenge..$^{[1,2]}$ These neoplasms range from enchondromas to chondrosarcomas with diagnostic margin being rather vague, particularly between enchondromas and low-grade chond rosarcomas where misdiagnosis may bring burdensome consequences. Currently, clinical discrimination mainly relies on location, radiologic, and pathologic properties of the tumor which yield little agreement between different clinicians for each patient. The need for a reliable and easily generalizable criteria is evident; however, there are no specific biomarkers available in the clinical setting despite ongoing studies. ${ }^{[3,4]}$

Recent decade has seen many reports delivering evidence on the role of inflammation in the development and carcinogenic advancement of neoplasms, although pathways remain mainly unknown up to this date. ${ }^{[5,6]}$ Therefore, blood-based markers of inflammation such as inflammatory cell counts and rates which are derived from those,

Received: January 20, 2020

Accepted: March 02, 2020

Published online: June 18, 2020

Correspondence: Aliekber Yapar, MD. Dr. Abdurrahman Yurtaslan Ankara Onkoloji Eğitim ve Araştırma Hastanesi Ortopedi ve Travmatoloji Kliniği, 06200 Yenimahalle, Ankara, Türkiye.

E-mail: aliekberyapar@hotmail.com

Doi: $10.5606 /$ ehc. 2020.73629

Citation: Yapar A, Ulucaköy C, Sezgin EA, Atalay İ, Ekșioğlu MF. Diagnostic role of neutrophil-to-lymphocyte ratio and monocyteto-lymphocyte ratio in patients with enchondroma and lowgrade chondrosarcoma. Jt Dis Relat Surg 2020;31(2):286-290.

\section{ABSTRACT}

Objectives: This study aims to evaluate the role of elevated neutrophil-to-lymphocyte ratio (NLR) and monocyte-tolymphocyte ratio (MLR) in differential diagnosis of enchondroma and low-grade chondrosarcoma.

Patients and methods: One-hundred-and-one patients (44 males, 57 females; mean age $53.6 \pm 11.5$ years; range, 21 to 85 years) diagnosed with enchondroma and low-grade chondrosarcoma in Ankara Oncology Training and Research Hospital between January 2010 and December 2019 were included in this retrospective study. Patients' age, gender, location and type of tumor, and pre-treatment complete blood count results were acquired. One-hundred patients (48 males, 52 females; mean age 50.9 \pm 13.6 years; range, 19 to 76 years) with complete blood count results admitted to the same center for reasons other than fracture, infection or tumors with similar age and gender to the aforementioned study group were included as healthy controls.

Results: Neutrophil-to-lymphocyte ratio and MLR of the study group were found to be significantly higher than the control group $(\mathrm{p}<0.001)$. Neutrophil-to-lymphocyte ratio and MLR held diagnostic importance with statistically significant cut-off values. Statistically significant cut-offs for NLR and MLR were $\geq 2.0$ (sensitivity $=73.3 \%$, specificity $=67 \%$ ) and $\geq 0.2$ (sensitivity $=76.2 \%$, specificity $=63 \%$ ), respectively. Multivariate logistic regression analysis was performed adjusting for age and gender and $\mathrm{NLR} \geq 2$ [odds ratio $(\mathrm{OR})=3.1$ ] or MLR $\geq 0.2(\mathrm{OR}=2.9)$ were found to be associated with approximately three-fold risk for diagnosis of enchondroma or low-grade chondrosarcoma.

Conclusion: The NLR and MLR have diagnostic value in cartilaginous tumors such as enchondroma and low-grade chondrosarcoma. However, our results do not support utilization of NLR and MLR as diagnostic value for differentiation of enchondroma and low-grade chondrosarcoma.

Keywords: Enchondroma, low-grade chondrosarcoma, monocyte-tolymphocyte ratio, neutrophil-to-lymphocyte ratio.

such as neutrophil-to-lymphocyte ratio (NLR) and monocyte-to-lymphocyte ratio (MLR), are being studied extensively not only in cancers of 
musculoskeletal system but other systems as well. ${ }^{[7-12]}$ Relying on current data and considering they can be easily obtained from routine complete blood counts, it is plausible to assume that NLR and MLR might have a previously unknown role in differential diagnosis of cartilaginous tumors. Therefore, in this study, we aimed to evaluate the role of elevated NLR and MLR in differential diagnosis of enchondroma and low-grade chondrosarcoma.

\section{PATIENTS AND METHODS}

Patients diagnosed with enchondroma and lowgrade chondrosarcoma in Ankara Oncology Training and Research Hospital between January 2010 and December 2019 were included in this retrospective study. One-hundred-and-one patients (44 males, 57 females; mean age $53.6 \pm 11.5$ years; range, 21 to 85 years) were identified in the institutional patient database and age, gender, location and type of tumor, and pretreatment complete blood count results were acquired retrospectively. Of 101 identified patients, 81 were diagnosed with enchondroma and 20 with low-grade

\begin{tabular}{|c|c|c|}
\hline \multicolumn{3}{|c|}{ TABLE I } \\
\hline Characteristic & $\mathrm{n}$ & $\%$ \\
\hline \multicolumn{3}{|l|}{ Pathology } \\
\hline Enchondroma & 81 & 80.2 \\
\hline Chondrosarcoma & 20 & 19.8 \\
\hline \multicolumn{3}{|l|}{ Localization } \\
\hline Enchondroma $(n=81)$ & 49 & 60.5 \\
\hline Distal femur & 32 & 39.5 \\
\hline \multicolumn{3}{|l|}{ Proximal humerus } \\
\hline Chondrosarcoma $(n=20)$ & 10 & 50 \\
\hline Distal femur & 10 & 50 \\
\hline Proximal humerus & & \\
\hline
\end{tabular}

chondrosarcoma. One-hundred patients (48 males, 52 females; mean age $50.6 \pm 13.6$ years; range, 19 to 76 years) with complete blood count results admitted to the same center for reasons other than fracture, infection or tumors with similar age and gender to the aforementioned study group were included as healthy controls. Patients without necessary information or with high C-reactive protein or procalcitonin were excluded. Neutrophil-to-lymphocyte ratio and MLR were calculated as the absolute count of neutrophils and monocyte, respectively, divided by the absolute lymphocyte count. The study protocol was approved by the Ankara Oncology Training and Research Hospital institutional review board. A written informed consent was obtained from each patient. The study was conducted in accordance with the principles of the Declaration of Helsinki.

\section{Statistical analysis}

Statistical analyses were performed using the IBM SPSS Statistics for Windows, version 22.0 software (IBM Corp., Armonk, NY, USA). Descriptive statistics are presented as numbers and percentages for categorical variables and mean \pm standard deviation, median (minimum-maximum) for continuous variables. Normal distribution for continuous variables was assessed with visual (histograms and probability graphics) and analytic methods (Kolmogorov-Smirnov and Shapiro-Wilk tests). Data between groups for continuous variables were found to be not fitting to normal distribution. Comparison analyses between two groups were performed by Mann-Whitney U test. Comparison analyses for categorical variables between independent groups were performed by chisquare test. Diagnostic value of pre-treatment NLR and MLR were assessed using receiver operating curve (ROC) analysis. Results following ROC analysis; area under curve and cut-off values, sensitivity and

\begin{tabular}{|c|c|c|c|c|c|c|c|c|c|c|c|}
\hline \multicolumn{12}{|c|}{$\begin{array}{l}\text { TABLE II } \\
\text { Evaluation of patient and control groups }\end{array}$} \\
\hline & \multicolumn{5}{|c|}{ Patients $(n=101)$} & \multicolumn{5}{|c|}{ Controls $(n=100)$} & \multirow[b]{2}{*}{$p$} \\
\hline & $\mathrm{n}$ & $\%$ & Mean $\pm S D$ & Median & Min-Max & $\mathrm{n}$ & $\%$ & Mean $\pm S D$ & Median & Min-Max & \\
\hline Age (year) & & & $53.6 \pm 11.5$ & 53 & $21-85$ & & & $50.9 \pm 13.6$ & 54 & $19-76$ & $0.279^{*}$ \\
\hline Gender & & & & & & & & & & & $0.528 \dagger$ \\
\hline Female & 57 & 56.4 & & & & 52 & 52.0 & & & & \\
\hline Male & 44 & 43.6 & & & & 48 & 48.0 & & & & \\
\hline NLR & & & $2.7 \pm 1.2$ & 2.54 & $1.12-7.46$ & & & $1.9 \pm 0.7$ & 1.75 & $0.75-3.68$ & $<0.001^{*}$ \\
\hline MLR & & & $0.3 \pm 0.1$ & 0.26 & $0.10-0.62$ & & & $0.2 \pm 0.1$ & 0.17 & $0.06-0.51$ & $<0.001^{*}$ \\
\hline
\end{tabular}




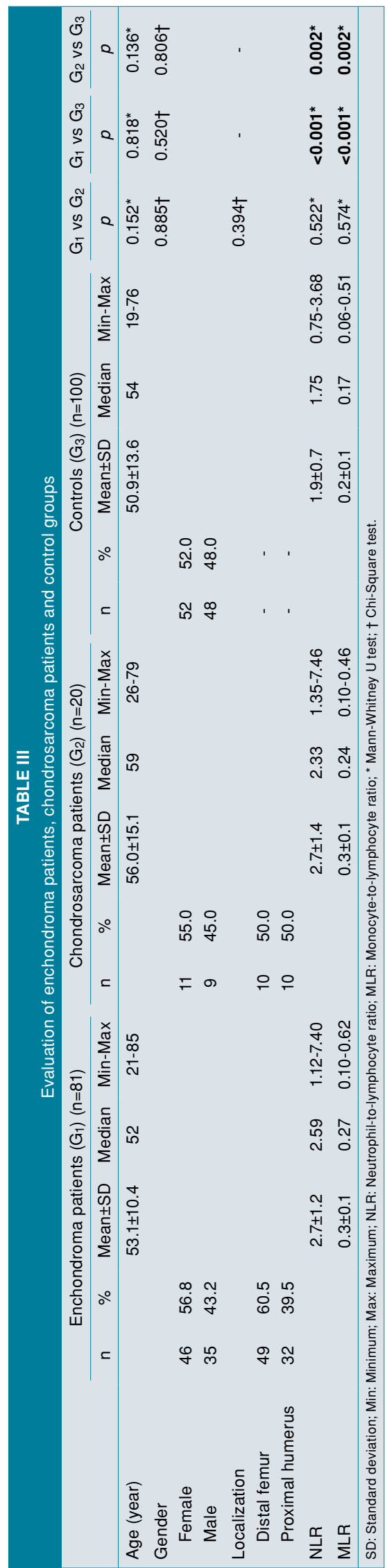

specificity of these cut-offs values, and positive and negative predictive values are presented. Effect of NLR and MLR on diagnosis was evaluated with multivariate logistic regression. Results of these analyses are presented as odds ratios (ORs) with 95\% confidence intervals (CIs). $\mathrm{P}$ values of $<0.05$ were considered to be statistically significant.

\section{RESULTS}

Of 101 identified patients, 81 were diagnosed with enchondroma and 20 with low-grade chondrosarcoma. Distal femur was the most common location for enchondromas $(n=49)$, followed by proximal humerus $(n=32)$. Half of the chondrosarcomas were located in distal femur and half in proximal humerus (Table I). No difference was observed for gender and age between study and control groups (Table II). Neutrophil-to-lymphocyte ratio and MLR of the study group were found to be significantly higher than the control group $(p<0.001)$ (Table II). However, no statistically significant difference was found for NLR and MLR values between enchondroma and low-grade chondrosarcoma groups $(\mathrm{p}=0.522$, $\mathrm{p}=0.574$, respectively). Neutrophil-to-lymphocyte ratio and MLR were significantly elevated in both enchondroma and chondrosarcoma groups compared to control group $(\mathrm{p}<0.001, \mathrm{p}=0.002$, respectively) (Table III).

Presence of a statistically significant cut-off value in NLR and MLR was evaluated with ROC analysis. This showed that NLR and MLR held diagnostic importance with statistically significant cut-off values (Table IV). Results of ROC analysis are presented in Figure 1. Statistically significant cut-offs for NLR and MLR were $\geq 2.0$ (sensitivity $=73.3 \%$, specificity $=67 \%$ ) and $\geq 0.2$ (sensitivity $=76.2 \%$, specificity $=63 \%$ ), respectively. Cut-offs for NLR and MLR defined by ROC analysis were used to categorize patients in the study and control groups. Multivariate logistic regression analysis was performed adjusting for age and gender and $N L R \geq 2(\mathrm{OR}=3.1)$ or $M L R \geq 0.2(\mathrm{OR}=2.9)$ were found to be associated with approximately three-fold risk for diagnosis of enchondroma or lowgrade chondrosarcoma (Table V).

\section{DISCUSSION}

Our study showed that pre-treatment NLR and MLR are tripled in enchondroma and low-grade chondrosarcoma patients compared to matched controls although it does not support use of NLR and MLR on determining the aggressiveness of cartilaginous bone tumors. ${ }^{[13]}$ 


\section{TABLE IV}

Statistical parameters of various diagnostic approaches for predictive value of preoperative neutrophil-to-lymphocyte ratio and monocyte-to-lymphocyte ratio

\begin{tabular}{lcccccccc} 
Parameter & AUC $(95 \% \mathrm{Cl})$ & $p$ & Cut-off & Sensitivity (\%) & Specificity (\%) & PPV (\%) & NPV (\%) \\
\hline NLR & $0.740(0.672-0.809)$ & $<0.001$ & $\geq 2$ & 73.3 & 67 & 69.2 & 71.3 \\
MLR & $0.748(0.680-0.816)$ & $<0.001$ & $\geq 0.2$ & 76.2 & 63 & 67.5 & 72.4
\end{tabular}

AUC: Area under curve; Cl: Confidence interval; PPV: Positive predictive value; NPV: Negative predictive value; NLR: Neutrophil-to-lymphocyte ratio; MLR Monocyte-to-lymphocyte ratio.

Enchondroma is common and often diagnosed incidentally; however, despite described benign radiological and clinical findings, distinguishing these from the aggressive form, low-grade chondrosarcoma, requires high suspicion and careful approach. Even with modern diagnostic

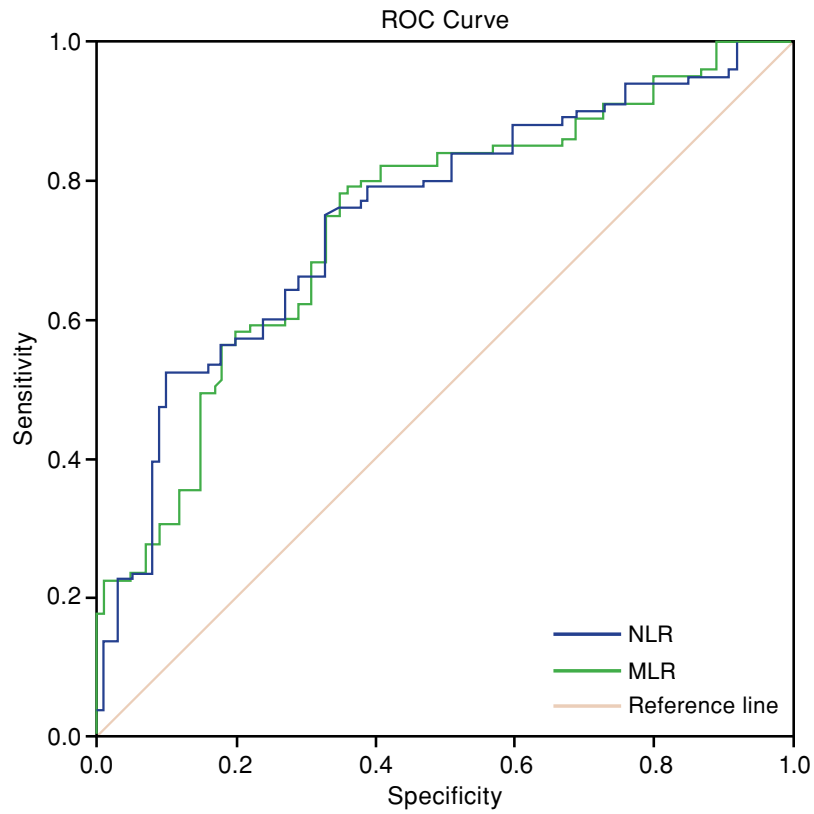

FIGURE 1. Receiver operating characteristic curves for NLR and MLR.

NLR: Neutrophil-to-lymphocyte ratio; MLR: Monocyte-to-lymphocyte ratio; ROC: Receiver operating characteristic.

\section{TABLE V}

Multivariate logistic regression analysis for NLR and MLR on enchondroma and chondrosarcoma

Multivariate logistic regression analysis model ${ }^{\star}$

Adjusted OR $(95 \% \mathrm{Cl})$ $p$

NLR $\geq 2$ (ref: $<2.25$ ) $3.1(1.5-6.5)$ 0.002 $M L R \geq 0.2$ (ref: $<0.2$ ) $2.9(1.4-6.1)$ 0.004

* Logistic regression analyses adjusted for gender and age; OR: Odds ratio; Cl: Confidence interval; NLR: Neutrophil-to-lymphocyte ratio; MLR: Monocyte-to-lymphocyte ratio. techniques such as bone scintigraphy and fluorodeoxyglucose positron emission tomographycomputed tomography, confident diagnosis remains a challenge..$^{[2]}$ Noninvasive biomarkers are often used in diagnosis of other neoplasms such as ovarian cancer, prostate cancer, gastric cancer, lung cancer, and many others. ${ }^{[14]}$ A similar approach to cartilaginous tumors of the skeleton would increase the much-needed diagnostic precision.

Systemic inflammatory biomarkers such as NLR and MLR in clinical management of neoplasms have recently started emerging as valid alternatives to conventional methods having been shown to correlate with diagnosis and/or prognosis in different kinds of tumors. ${ }^{[7-12]}$ Current interest in utilizing these ratios seems justified as these are readily available values derived from routine complete blood count with no economic burden.

A 2014 meta-analysis of over 40,000 solid malignancy patients showed that the median cutoff of 4 can be regarded as an indicator of worse outcome. ${ }^{[15]}$ Thio et al. ${ }^{[16]}$ and Wang et al. ${ }^{[17]}$ have found that 3 would be a reasonable cut-off value in estimating prognosis in cancer patients with bone metastases. We had a different approach where we aimed to shed light on current challenges in differential diagnosis of two cartilaginous tumors with differentaggressiveness. Theliterature suggests that a tumor with malignant characteristics would be associated with elevated neutrophils which secrete large amounts of reactive oxygen species and vascular endothelial growth factors that induce deoxyribonucleic acid damage, angiogenesis and promote tumor microenvironment. ${ }^{[18,19]}$ Neutrophils are also suggested to negatively influence T-cell activation which results in reduced lymphocyte-mediated immune response. ${ }^{[2,21]}$ In addition, increased number of tumor-associated macrophages, originating from circulating monocytes, has been shown to be correlated with malignant characteristics. ${ }^{[22]}$ In our study, the rates of NLR and MLR were significantly higher in the cartilaginous tumor groups such as enchondroma 
and low-grade chondrosarcoma compared to the control group. Our initial expectation was that chondrosarcoma would be associated with higher NLR and MLR which would enable differentiation from enchondroma. However, our study has shown no statistically significant difference between these tumors which may be due to complex inflammatory characteristics of cartilaginous tumors or the small sample size, which we acknowledge as a limitation. Another limitation would be the retrospective, single-center nature of the study. We believe that future studies with larger sample sizes are necessary to further explore the characteristics of inflammation and role of systemic inflammatory biomarkers in cartilaginous skeletal tumors.

In conclusion, NLR and MLR have diagnostic value in cartilaginous tumors such as enchondroma and low-grade chondrosarcoma. However, our results do not support utilization of NLR and MLR as diagnostic value for differentiation of enchondroma and low-grade chondrosarcoma.

\section{Declaration of conflicting interests}

The authors declared no conflicts of interest with respect to the authorship and/or publication of this article.

\section{Funding}

The authors received no financial support for the research and/or authorship of this article.

\section{REFERENCES}

1. Skeletal Lesions Interobserver Correlation among Expert Diagnosticians (SLICED) Study Group. Reliability of histopathologic and radiologic grading of cartilaginous neoplasms in long bones. J Bone Joint Surg [Am] 2007;89:211323.

2. Mulligan ME. How to Diagnose Enchondroma, Bone Infarct, and Chondrosarcoma. Curr Probl Diagn Radiol 2019;48:262-73.

3. Zhang L, Yang M, Mayer T, Johnstone B, Les C, Frisch $\mathrm{N}$, et al. Use of MicroRNA biomarkers to distinguish enchondroma from low-grade chondrosarcoma. Connect Tissue Res 2017;58:155-61.

4. Tamam C, Tamam M. Prognostic significance of maximum standardized uptake value on 18F-fluoro-2-deoxyglucose positron emission tomography/computed tomography in bone sarcomas. Eklem Hastalik Cerrahisi 2018;29:184-8.

5. Gregory AD, Houghton AM. Tumor-associated neutrophils: new targets for cancer therapy. Cancer Res 2011;71:2411-6.

6. Grivennikov SI, Greten FR, Karin M. Immunity, inflammation, and cancer. Cell 2010;140:883-99.
7. Gregory AD, Houghton AM. Tumor-associated neutrophils: new targets for cancer therapy. Cancer Res 2011;71:2411-6.

8. Shen J, Zhu Y, Wu W, Zhang L, Ju H, Fan Y, et al. Prognostic Role of Neutrophil-to-Lymphocyte Ratio in Locally Advanced Rectal Cancer Treated with Neoadjuvant Chemoradiotherapy. Med Sci Monit 2017;23:315-24.

9. Prodromidou A, Andreakos P, Kazakos C, Vlachos DE, Perrea D, Pergialiotis V. The diagnostic efficacy of plateletto-lymphocyte ratio and neutrophil-to-lymphocyte ratio in ovarian cancer. Inflamm Res 2017;66:467-75.

10. Caliskan B, Korkmaz AN. Can Neutrophil/Lymphocyte Ratio be a Predictor for Bone Metastases of Solid Tumors? World J Nucl Med 2016;15:196-9.

11. Liu G, Ke LC, Sun SR. Prognostic value of pretreatment neutrophil-to-lymphocyte ratio in patients with soft tissue sarcoma: A meta-analysis. Medicine (Baltimore) 2018;97:e12176.

12. Zhao G, Wang J, Xia J, Wei Y, Wang S, Huang G, et al. The predictive value of preoperative neutrophil-lymphocyte ratio (NLR) on the recurrence of the local pigmented villonodular synovitis of the knee joint. BMC Musculoskelet Disord 2018;19:339.

13. Atik OŞ. Which articles do we prefer to publish? Eklem Hastalik Cerrahisi 2018;29:1.

14. Duffy MJ. Use of Biomarkers in Screening for Cancer. Adv Exp Med Biol 2015;867:27-39.

15. Templeton AJ, McNamara MG, Šeruga B, Vera-Badillo FE, Aneja P, Ocaña A, et al. Prognostic role of neutrophil-tolymphocyte ratio in solid tumors: a systematic review and meta-analysis. J Natl Cancer Inst 2014;106:dju124.

16. Thio QCBS, Goudriaan WA, Janssen SJ, Paulino Pereira NR, Sciubba DM, Rosovksy RP, et al. Prognostic role of neutrophil-to-lymphocyte ratio and platelet-to-lymphocyte ratio in patients with bone metastases. $\mathrm{Br} \mathrm{J}$ Cancer 2018;119:737-43.

17. Wang S, Zhang Z, Fang F, Gao X, Sun W, Liu H. The neutrophil/lymphocyte ratio is an independent prognostic indicator in patients with bone metastasis. Oncol Lett 2011;2:735-40.

18. Kusumanto YH, Dam WA, Hospers GA, Meijer C, Mulder NH. Platelets and granulocytes, in particular the neutrophils, form important compartments for circulating vascular endothelial growth factor. Angiogenesis 2003;6:283-7.

19. Shamamian P, Schwartz JD, Pocock BJ, Monea S, Whiting D, Marcus SG, et al. Activation of progelatinase A (MMP-2) by neutrophil elastase, cathepsin $\mathrm{G}$, and proteinase-3: a role for inflammatory cells in tumor invasion and angiogenesis. J Cell Physiol 2001;189:197-206.

20. MüllerI,MunderM,KropfP,HänschGM.Polymorphonuclear neutrophils and $\mathrm{T}$ lymphocytes: strange bedfellows or brothers in arms? Trends Immunol 2009;30:522-30.

21. Ferrone C, Dranoff G. Dual roles for immunity in gastrointestinal cancers. J Clin Oncol 2010;28:4045-51.

22. Mantovani A, Schioppa T, Porta C, Allavena P, Sica A. Role of tumor-associated macrophages in tumor progression and invasion. Cancer Metastasis Rev 2006;25:315-22. 